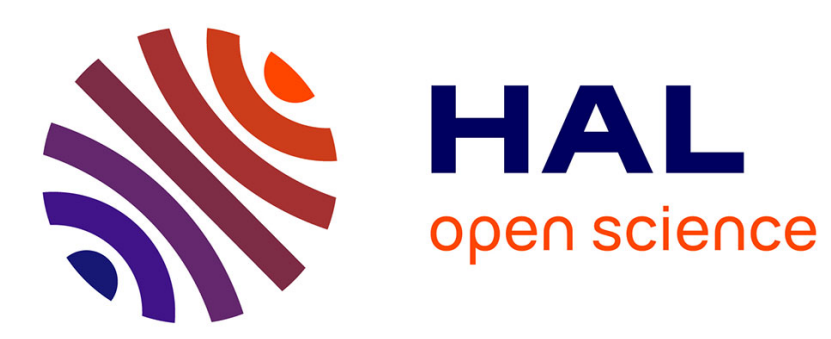

\title{
Optimal Time Interval Selection in Long-Run Correlation Estimation
}

Pedro Albuquerque

\section{To cite this version:}

Pedro Albuquerque. Optimal Time Interval Selection in Long-Run Correlation Estimation. Journal of Quantitative Economics, 2020, 18 (1), pp.53-79. 10.1007/s40953-019-00175-x . hal-02482675

\section{HAL Id: hal-02482675 \\ https://hal-amu.archives-ouvertes.fr/hal-02482675}

Submitted on 9 Jul 2020

HAL is a multi-disciplinary open access archive for the deposit and dissemination of scientific research documents, whether they are published or not. The documents may come from teaching and research institutions in France or abroad, or from public or private research centers.
L'archive ouverte pluridisciplinaire $\mathbf{H A L}$, est destinée au dépôt et à la diffusion de documents scientifiques de niveau recherche, publiés ou non, émanant des établissements d'enseignement et de recherche français ou étrangers, des laboratoires publics ou privés. 


\title{
Optimal Time Interval Selection in Long-Run Correlation Estimation
}

\author{
Pedro H. Albuquerque ${ }^{1,2}$ (D)
}

\begin{abstract}
This paper presents an asymptotically optimal time interval selection criterion for the long-run correlation block estimator (Bartlett kernel estimator) based on the NeweyWest and Andrews-Monahan approaches. An alignment criterion that enhances finite-sample performance is also proposed. The procedure offers an opti-mal alternative to the customary practice in finance and economics of heuristically or arbitrarily choosing time intervals or lags in correlation studies. A Monte Carlo experiment using parameters derived from Dow Jones returns data confirms that the procedure can be MSE-superior to alternatives such as aggregation over arbitrary time intervals, parametric VAR, and Newey-West covariance matrix estimation with automatic lag selection.
\end{abstract}

Keywords Long-run correlation · Bartlett · Lag selection · Alignment · Newey-West · Andrews-Monahan

JEL Classification C14 · C22

\section{Introduction}

Correlation measures are frequently used in economics and finance to characterize the relations between pairs of time series, many times as a prelude to more detailed empirical analyses. Estimation procedures however are usually nonoptimal. For exam-ple, in studies of the relation between stock returns, a typical approach is to apply a simple correlation estimator to weekly or monthly aggregate returns, even though data is usually available at higher frequencies. ${ }^{1}$ Another common procedure is the use of a

${ }^{1}$ Examples can be found in Campbell and Ammer (1993) and Ammer and Mei (1996).

$\bowtie \quad$ Pedro H. Albuquerque

pedro.albuquerque@kedgebs.com

1 KEDGE Business School, Domaine de Luminy, BP 921, 13288 Marseille, France

2 Aix-Marseille Univ., CNRS, EHESS, Centrale Marseille, IRD, AMSE, Marseille, France 
VAR to estimate correlation measures, where the number of lags is chosen arbitrarily or using an information criterion. ${ }^{2}$

Furthermore, time series correlation studies are many times concerned with permanent relations. For example, studies of stock market returns may want to characterize only the long-run relationship, filtering out the effects of reversible components. Long-run estimates tend to be heuristically approximated through the choice of return horizons or lags that are considered long enough to capture permanent effects. There are however two problems with this customary practice. When the procedure involves time aggregation of high-frequency data, there may be unnecessary loss of information. Additionally, there is usually no concern for the optimality of time interval or lag choices.

For instance, a researcher may have access to daily stock return data, and yet choose to use aggregate monthly return data to estimate return correlations. The procedure though is inefficient, since the aggregation of daily data into monthly data can be improved on by using an estimator based on overlapping monthly returns defined daily, in other words, a block estimator. Additionally, the choice of a monthly time interval is arbitrary and not necessarily optimal, particularly when the explicit goal of the analyst is the characterization of the relation between permanent components. For example, a monthly time interval may imply a return horizon that is or too short, and thus less able to filter out transitory components, or too long, and consequently less efficient.

One can propose therefore a better procedure that has the additional advantage of not significantly departing from common practice in time series correlation studies. The procedure employs a block estimator and an optimal time interval selection criterion, or, formally, it uses a nonparametric consistent long-run correlation estimator based on the $k$-lag difference correlation estimator (Bartlett kernel estimator) combined with automatic lag selection and alignment criteria. Notice that, in the case of the block estimator, the choice of a kernel size is equivalent to the choice of a time interval. This property is exactly what makes the procedure simple, convenient and familiar to practitioners not trained in spectral methods.

The methodological approach follows Andrews and Monahan (1992) and Newey and West (1994). The automatic lag selection criterion is based on the minimization of the asymptotic MSE, leading to a time interval choice that is long enough to minimize the estimator bias and short enough to minimize the estimator variance.

It may appear to some that an optimal criterion for long-run correlation estimation is unnecessary, since the Newey-West procedure can be used to optimally estimate longrun covariance matrices and, therefore, to calculate long-run correlations. However, the Newey-West procedure does not guarantee the optimal estimation of correlation, so a new criterion is designed to minimize instead the MSE of long-run correlation.

A Monte Carlo experiment using a VMA (5) and GARCH $(1,1)$ parameters derived from Dow Jones returns data is used to evaluate the effectiveness of the lag selection and alignment criteria. The proposed estimator proves to be adequate and MSE-superior to commonly employed alternatives.

\footnotetext{
${ }^{2}$ See for example King and Watson (1994) and Forbes and Rigobon (2002).
} 


\section{Long-Run Correlation}

Long-run correlation can be defined using the concept of complex coherency from spectral analysis. ${ }^{3}$ Following Priestley (1981), given two random variables $x_{t}$ and $y_{t}$, the coherency at frequency $\omega$ can be interpreted as the correlation between the random coefficients of the spectral components of $x_{t}$ and $y_{t}$ at frequency $\omega$ :

$$
C(\omega)=\frac{s_{x y}(\omega)}{\sqrt{s_{x x}(\omega) s_{y y}(\omega)}},|C(\omega)| \leq 1,\left|s_{x x}(\omega)\right|>0,\left|s_{y y}(\omega)\right|>0,
$$

where $s_{x x}(\omega)$ and $s_{y y}(\omega)$ are the spectra and $s_{x y}(\omega)$ is the cross-spectrum, or, in other words, the Fourier transforms of autocovariances and cross-covariances, given by

$$
\begin{aligned}
& s_{x x}(\omega)= \frac{1}{2 \pi} \sum_{n=-\infty}^{\infty} \gamma_{x x}(n) e^{-i \omega n}, \quad s_{y y}(\omega) \\
&=\frac{1}{2 \pi} \sum_{n=-\infty}^{\infty} \gamma_{y y}(n) e^{-i \omega n}, \quad s_{x y}(\omega)=\frac{1}{2 \pi} \sum_{n=-\infty}^{\infty} \gamma_{x y}(n) e^{-i \omega n}, \\
& \gamma_{x x}(n)=E\left[\left(x_{t}-\mu_{x}\right)\left(x_{t-n}-\mu_{x}\right)\right], \quad \gamma_{y y}(n)=E\left[\left(y_{t}-\mu_{y}\right)\left(y_{t-n}-\mu_{y}\right)\right], \\
& \gamma_{x y}(n)=E\left[\left(x_{t}-\mu_{x}\right)\left(y_{t-n}-\mu_{y}\right)\right], \quad \mu_{x}=E\left[x_{t}\right], \quad \mu_{y}=E\left[y_{t}\right],
\end{aligned}
$$

where $\gamma$ represents the autocovariances or the cross-covariances of $x_{t}$ and $y_{t} .{ }^{4}$

The time-domain concept of long-run correlation, which applies to pairs of $I(0)$ variables, is equivalent to the frequency-domain concept of complex coherency at frequency zero $(\omega=0)$,

$$
\lambda \equiv \frac{s_{x y}}{\sqrt{s_{x x} s_{y y}}}, \quad-1 \leq \lambda \leq 1, \quad s_{x x}>0,
$$

where

$$
s_{x y}=\frac{1}{2 \pi} \sum_{n=-\infty}^{\infty} \gamma_{x y}(n), \quad s_{x x}=\frac{1}{2 \pi} \sum_{n=-\infty}^{\infty} \gamma_{x x}(n), \quad s_{y y}=\frac{1}{2 \pi} \sum_{n=-\infty}^{\infty} \gamma_{y y}(n) .
$$

Notice that if $x_{t}$ and $y_{t}$ are overdifferenced (the first differences of $I(0)$ variables) then the long-run correlation parameter $\lambda$ is not defined, since $s_{x x}$ and $s_{y y}$ are equal to zero. In other words, overdifferencing has the effect of a high-pass filter, eliminating all long-run information from a variable.

Granger and Weiss (1983) and Engle and Granger (1987) observed that, under certain cointegration literature assumptions, two $I(1)$ variables cointegrate if and only if their first differences have squared long-run correlation equal to one. On the other

\footnotetext{
3 See for example Granger and Rees (1968), Granger and Engle (1983) and McCallum (1984).

${ }^{4}$ See Anderson (1971), Koopmans (1974), Fuller (1976), Priestley (1981), Granger and Watson (1984) and Brockwell and Davis (1991).
} 
hand, zero long-run correlation between first differences will only imply the absence of structural long-run relation between two $I(1)$ variables under certain identification restrictions, see for example Fisher and Seater (1993). Notice also that the fact that two variables do not cointegrate does not imply the absence of structural long-run relationship; see for example McCallum (1993) for a discussion. Moreover, under certain identification assumptions, a nonzero long-run correlation value will indicate the existence of structural long-run relationship, even in the absence of cointegration. Fisher and Seater (1993) offer an example using VARMA models.

\section{Nonparametric Long-Run Granger Causality}

Time series empirical studies have frequently relied on the concept of Granger causality as part of their analytical toolbox, see for example Nachane et al. (1988) for a discussion with applications. Granger causality is commonly studied using parametric tests and linear autoregressive models under the null hypothesis of non-causality, but nonparametric approaches are also available, such as in Diks and Panchenko (2006).

The concept of coherency is known to be useful in the evaluation of Granger causality. According to Granger (1969), and using that article's terminology, the crossspectrum function $s_{x y}(\omega)$ as defined in (1) can be decomposed into three parts

$$
s_{x y}(\omega)=s_{\overrightarrow{x y}}(\omega)+s_{\overrightarrow{y x}}(\omega)+s_{\widetilde{x y}}(\omega),
$$

which can be used to define the following causality-coherency expressions:

$$
C_{\overrightarrow{x y}}(\omega)=\frac{s_{\overrightarrow{x y}}(\omega)}{\sqrt{s_{x x}(\omega) s_{y y}(\omega)}}, \quad C_{\overrightarrow{y x}}(\omega)=\frac{s_{\overrightarrow{y x}}(\omega)}{\sqrt{s_{x x}(\omega) s_{y y}(\omega)}}, \text { and } \quad C_{\widetilde{y x}}(\omega)=\frac{s_{\widetilde{x y}}(\omega)}{\sqrt{s_{x x}(\omega) s_{y y}(\omega)}},
$$

where $C_{\overrightarrow{x y}}(\omega)$ measures strength of causality from $x$ to $y$ at frequency $\omega, C_{\overrightarrow{y x}}(\omega)$ does the same for $y$ to $x$, and $C_{\widetilde{x y}}(\omega)$ measures instantaneous causality between $x$ and $y$.

Equivalent long-run causality-coherency expressions can be defined by making $\omega$ equal to zero:

$$
\lambda_{\overrightarrow{x y}}=\frac{s_{\overrightarrow{x y}}}{\sqrt{s_{x x} s_{y y}}}, \quad \lambda_{\overrightarrow{y x}}=\frac{s_{\overrightarrow{y x}}}{\sqrt{s_{x x} s_{y y}}}, \text { and } \quad \lambda_{\widetilde{y x}}=\frac{s_{\widetilde{x y}}}{\sqrt{s_{x x} s_{y y}}} .
$$

The concept of long-run Granger causality is of interest in economics, see for example Granger and Lin (1995) for an in-depth discussion of the subject. Additional exploration of this topic, such as the construction of appropriate tests for the null hypothesis that causality-coherency expressions are not statistically different from zero, are potential extensions of this article.

\section{Nonparametric Estimation of Long-Run Correlation}

This section presents a nonparametric estimator of long-run correlation based on the block estimator ( $k$-lag difference) approach of Bartlett (1950), Cochrane (1988) and 
Cochrane and Sbordone (1988). Cochrane for example presented a nonparametric statistic for unit root processes called the variance ratio, based on the $k$-lag difference variance of a series. This paper goes a few steps further in this line of research by developing automatic time interval selection and alignment criteria for the long-run correlation block estimator.

\section{Estimator}

Consider two random variables $x_{t}$ and $y_{t}$ with summable covariances and autocovariances. Given a sample of size $T, 1 \leq t \leq T$, an analogue estimator $\hat{\lambda}_{b}$ of the long-run correlation defined in (2) is a kernel estimator

$$
\hat{\lambda}_{b}=\frac{\hat{s}_{x y}(b)}{\sqrt{\hat{s}_{x x}(b) \hat{s}_{y y}(b)}},
$$

where the cross-spectrum and spectrum estimators are

$$
\begin{array}{r}
\hat{s}_{x y}(b)=\frac{1}{2 \pi} \sum_{n=-T+1}^{T-1} \kappa(n, b) \hat{\gamma}_{x y}(n), \quad \hat{s}_{x x}(b)=\frac{1}{2 \pi} \sum_{n=-T+1}^{T-1} \kappa(n, b) \hat{\gamma}_{x x}(n), \\
\hat{s}_{y y}(b)=\frac{1}{2 \pi} \sum_{n=-T+1}^{T-1} \kappa(n, b) \hat{\gamma}_{y y}(n),
\end{array}
$$

the cross-covariance and autocovariance estimators are

$$
\begin{gathered}
\hat{\gamma}_{x y}(n)=\left\{\begin{array}{c}
\frac{1}{T} \sum_{t=1}^{T+n}\left(x_{t}-\bar{\mu}_{x}\right)\left(y_{t-n}-\bar{\mu}_{y}\right), \quad n<0, \\
\frac{1}{T} \sum_{t=1}^{T-n}\left(x_{t+n}-\bar{\mu}_{x}\right)\left(y_{t}-\bar{\mu}_{y}\right), \quad n \geq 0,
\end{array}\right. \\
\hat{\gamma}_{x x}(n)=\frac{1}{T} \sum_{t=1}^{T-|n|}\left(x_{t+|n|}-\bar{\mu}_{x}\right)\left(x_{t}-\bar{\mu}_{x}\right), \quad \bar{\mu}_{x}=\frac{1}{T} \sum_{t=1}^{T} x_{t}, \\
\hat{\gamma}_{y y}(n)=\frac{1}{T} \sum_{t=1}^{T-|n|}\left(y_{t+|n|}-\bar{\mu}_{y}\right)\left(y_{t}-\bar{\mu}_{y}\right), \quad \bar{\mu}_{y}=\frac{1}{T} \sum_{t=1}^{T} y_{t},
\end{gathered}
$$

and $\kappa(n, b)$ is a kernel with bandwidth $b, 1 \leq b \leq T-1 .^{5}$

Different kernels can be used, each one having advantages and disadvantages, as discussed in Newey and West $(1987,1994)$ and Andrews (1991). The Newey-West

\footnotetext{
5 See Priestley (1981, pg. 432). In spectral matrix estimation, the kernel parameter $b$ can be a real number, but here the analysis will limit itself to integer values of $b$ to enforce compatibility of the Bartlett kernel spectral estimator with the discrete-time version of the block estimator presented later in this subsection.
} 
(Bartlett) kernel approach is chosen in this paper, due to its block estimator equivalence, as discussed in what follows. ${ }^{6}$

Consider the expression for the covariance block estimator ( $k$-lag difference estimator) $\hat{\sigma}_{X Y}(k)$ divided by $k$, where, as in Cochrane (1988), the substitution of $k$ times the average of the first differences for the average of the $k$-lag differences is used as a finite-sample enhancement:

$$
\begin{array}{r}
\frac{\hat{\sigma}_{X Y}(k)}{k}=\frac{1}{k} \sum_{t=k}^{T} \frac{\left[\left(1-L^{k}\right) X_{t}-k \bar{\mu}_{x}\right]\left[\left(1-L^{k}\right) Y_{t}-k \bar{\mu}_{y}\right]}{T-k}, \\
\left(1-L^{k}\right) X_{t}=X_{t}-X_{t-k}, \quad\left(1-L^{k}\right) Y_{t}=Y_{t}-Y_{t-k},
\end{array}
$$

where $X_{t}$ and $Y_{t}$ are the integrated versions of $x_{t}$ and $y_{t}$, given by

$$
X_{t}=X_{0}+\sum_{i=1}^{t} x_{i}, \quad Y_{t}=Y_{0}+\sum_{j=1}^{t} y_{j}, \text { for } 1 \leq t \leq T
$$

As shown in "Appendix 1", the block estimator with a time-interval parameter $k$ converges in probability to $2 \pi$ times the Bartlett kernel estimator with bandwidth $b=$ $k^{7}$ :

$$
\frac{\hat{\sigma}_{X Y}(k)}{k}=\frac{T}{T-k} 2 \pi \hat{s}_{x y}(k)+O_{p}\left(\frac{k}{T}\right),
$$

and the correlation block estimator $\hat{\Lambda}_{k}$ can be used therefore to estimate long-run correlation instead of Eq. (4), holding the same asymptotic properties:

$$
\hat{\Lambda}_{k}=\frac{\hat{\sigma}_{X Y}(k)}{\sqrt{\hat{\sigma}_{X X}(k) \hat{\sigma}_{Y Y}(k)}} .
$$

To confirm the adequacy of this result in the case of finite samples, a comparison of the spectral (4) and the block (5) estimator using a Monte Carlo simulation will be provided in "Monte Carlo Simulations".

Notice that, from this point on, and for notational convenience, the use of the parameter $k$ instead of the parameter $b$ in spectral estimators indicates the use of a Bartlett kernel with bandwidth $b=k$.

\footnotetext{
6 Andrews (1991) proved that the QS kernel is optimal with respect to an asymptotic truncated MSE criterion among kernels that generate positive semi-definite covariance estimates, such as the Bartlett kernel. This result however should not be definitive evidence against the use of the Bartlett kernel, since the latter may perform better than the QS kernel with finite samples, as discussed in Andrews (1991) and as shown in Newey and West (1994). Newey and West argue for example that the Bartlett kernel performs better when processes are characterized by autocorrelations that "die out slowly"-a case frequently found in economic data, see for example Cochrane (1988). Moreover, as discussed in Hannan (1970, pg. 287), the QS kernel, differently from the Bartlett kernel, assumes negative values, leading to long-run correlation estimates that are not bounded (in absolute value) by unity, a property that, besides being undesirable by itself, complicates the derivation of the statistical properties of the estimator.
}

7 See also Cochrane (1988). 
The block estimator has convenient features: it is a simple correlation estimator applied to changes measured over time intervals of size $k$. Despite its straightforward time-domain representation, its asymptotic properties can be derived using frequencydomain methods. Its estimates are identical to those of heuristic procedures widely adopted in different fields of economics and finance if the same time interval is chosen. Because of this property, the block estimator provides the practitioner with a familiar and intuitive interpretation of its optimal parameters. Therefore, preference will be given to the block estimator in the sections that follow, always keeping in mind that the Bartlett kernel estimator can be used instead if preferred.

Practitioners have used similar yet less efficient procedures when heuristically or arbitrarily selecting time intervals or lags and when aggregating high-frequency data into longer intervals, perhaps unaware of the statistical implication of their choices. The block estimator proposed here, as will be shown in the next subsections, is therefore an optimal yet unobtrusive alternative to commonly employed methods.

\section{Consistency}

As shown in Andrews (1991), "automatic bandwidth kernel estimators are consistent with nonstationary as well as fourth order stationary random variables". Apply now a Taylor expansion around $s_{x x}, s_{y y}$, and $s_{x y}$ to the proposed long-run correlation estimator:

$$
\hat{\lambda}_{k}=\lambda+\boldsymbol{D}_{\lambda}\left[\begin{array}{c}
\hat{s}_{x x}(k)-s_{x x} \\
\hat{s}_{y y}(k)-s_{y y} \\
\hat{s}_{x y}(k)-s_{x y}
\end{array}\right]+O_{p}\left(\left(\frac{k^{2}}{T}\right)^{\frac{1}{2}}\right)
$$

where

$$
\boldsymbol{D}_{\lambda}=\left[\frac{\partial \lambda}{\partial s_{x x}} \frac{\partial \lambda}{\partial s_{y y}} \frac{\partial \lambda}{\partial s_{x y}}\right]=\left[\frac{-\lambda}{2 s_{x x}} \frac{-\lambda}{2 s_{y y}} \frac{1}{\sqrt{s_{x x} S_{y y}}}\right] .
$$

The Taylor expansion shows that the long-run correlation estimator converges in probability to a linear combination of consistent spectral matrix estimators at frequency zero, meaning that the Bartlett kernel estimator of long-run correlation is also consistent under the same conditions of Andrews (1991).

\section{Lag Selection}

The following MSE-minimization procedure will be valid under the set of assumptions given by Andrews (1991). Using the same approach of Newey and West (1994), an optimal lag selection criterion minimizes the asymptotic MSE (AMSE) of the longrun correlation estimator by exploiting the trade-off between the asymptotic variance (Avar), obtained in "Appendix 2",

$$
\operatorname{Avar}\left(\hat{\lambda}_{k}\right)=\frac{2}{3} \frac{k}{T}\left(1-\lambda^{2}\right)^{2},
$$


and the square of the asymptotic bias (Abias), obtained in "Appendix 3",

$$
\operatorname{Abias}\left(\hat{\lambda}_{k}\right)^{2}=\frac{\psi^{2}}{k^{2}},
$$

where

$$
\begin{gathered}
\psi=\frac{s_{x y}^{(1)}}{\sqrt{s_{x x} s_{y y}}}-\frac{\lambda}{2}\left(\frac{s_{x x}^{(1)}}{s_{x x}}+\frac{s_{y y}^{(1)}}{s_{y y}}\right), \\
s_{x x}^{(1)}=\frac{1}{2 \pi} \sum_{n=-\infty}^{\infty}|n| \gamma_{x x}(n), \quad s_{y y}^{(1)}=\frac{1}{2 \pi} \sum_{n=-\infty}^{\infty}|n| \gamma_{y y}(n),
\end{gathered}
$$

and

$$
s_{x y}^{(1)}=\frac{1}{2 \pi} \sum_{n=-\infty}^{\infty}|n| \gamma_{x y}(n)
$$

The following proposition is proved in "Appendix 4":

Proposition 1 when $\lambda^{2}<1$, the lag selection criterion that minimizes the asymptotic mean squared error of the Bartlett kernel long-run correlation estimator is

$$
k^{*}=\left\lceil 1.4422\left[\left(\frac{\psi}{1-\lambda^{2}}\right)^{2} T\right]^{\frac{1}{3}}\right]
$$

where $\lceil\cdot\rceil$ is the integer ceiling function.

Notice that Proposition 1 is not a particular case of the Newey and West (1994) automatic lag selection criterion. The Newey-West procedure is based on the minimization of the MSE of a weighted function of the estimated spectral matrix $\hat{\boldsymbol{S}}(k)$ :

$$
\min E\left[\boldsymbol{w}^{\prime}(\hat{\boldsymbol{S}}(k)-\boldsymbol{S}) \boldsymbol{w}\right]^{2}
$$

where

$$
\boldsymbol{S}=\left[\begin{array}{ll}
s_{x x} & s_{x y} \\
s_{y x} & s_{y y}
\end{array}\right] \text { and } \hat{\mathbf{S}}(k)=\left[\begin{array}{ll}
\hat{s}_{x x}(k) & \hat{s}_{x y}(k) \\
\hat{s}_{y x}(k) & \hat{s}_{y y}(k)
\end{array}\right]
$$

And where $\mathbf{w}$ is a weight vector. Proposition 1, on the other hand, is based on the minimization of the MSE of the long-run correlation function:

$$
\min E\left[{ }_{k}-\lambda\right]^{2}
$$

These two MSE cannot be rendered equivalent, since there is no choice of $\mathbf{w}$ that can simultaneously solve $\boldsymbol{w} \hat{\boldsymbol{S}}(k) \boldsymbol{w}=\hat{\lambda}_{k}$ and $\boldsymbol{w} \boldsymbol{S} \boldsymbol{w}=\lambda$. The Newey-West lag selection 
procedure therefore cannot be used to optimally estimate long-run correlation, even though it can be used to optimally estimate long-run covariance matrices. This result will be confirmed in "Monte Carlo Simulations" through a Monte Carlo simulation using, among other procedures, a standard Newey-West estimator with a weight vector of ones.

\section{Alignment}

In some cases, a time shift of the kernel of one variable in relation to another-a procedure called alignment-may improve the finite-sample properties of a crossspectrum estimator. ${ }^{8}$ The use of an alignment procedure is particularly useful when the analyst believes that one of the variables "leads or lags" the other variable in the time dimension.

For example, suppose that a practitioner wants to study the relationship between money and inflation using monthly data under the prior that money "leads" inflation by 18 months. The practitioner may be expressing a belief that the correlation between money and inflation is strongest when changes in money that happen today are correlated with changes of inflation that happen 18 months from now. In the context of alignment procedures, this prior can serve as the centre point of a search range for the alignment parameter.

In the case of the block estimator, time-shifting the kernel is equivalent to lagging one variable in relation to another. In the example above, the practitioner may lag money by 18 months and "align" it with inflation before estimating correlation.

Define therefore the alignment parameter $a^{*}$ as follows.

Definition 1 The alignment parameter $a^{*}$ is equal to the value of a that minimizes the following weighted sum of absolute covariances:

$$
a^{*}=\underset{a_{\min } \leq a \leq a_{\max }}{\arg \min } \sum_{n=a_{\min }}^{a_{\max }}|n-a| \cdot\left|\gamma_{x y}(n)\right|, \quad\left|a_{\min }\right|<\infty, \quad\left|a_{\max }\right|<\infty, \quad a_{\min } \leq a_{\max } .
$$

The parameters $a^{*}, a_{\min }$ and $a_{\max }$ are integer numbers, where $a^{*}$ represents the kernel shift alignment parameter and $a_{\min }$ and $a_{\max }$ represent the chosen boundaries of the search range. Notice that the lack of an alignment criterion is equivalent to the restrictive assumption that the alignment parameter search runs from zero to zero.

The alignment parameter is used to relocate the cross-spectrum kernel such that the highest kernel weights are applied to the cross-covariances with the highest absolute values, typically improving the finite-sample performance of the estimator. This statement can be intuitively verified in the case of the long-run correlation Bartlett kernel estimator by noticing that the alignment procedure "synchronizes" the two series, what leads to better finite-sample estimates of $s_{x y}$ that, in their turn, improve the finite-sample estimate of $\lambda$.

\footnotetext{
${ }^{8}$ See Priestley (1981, pg. 710).
} 
Notice that the alignment technique is a heuristic procedure not based on asymptotic theory, and also that this technique does not change the asymptotic properties of the estimator. On the other hand, as Monte Carlo simulations will show in "Monte Carlo Simulations", it can improve finite-sample performance of the long-run correlation estimator when "misalignments" are present, sometimes by substantial margins.

\section{Lag Selection and Alignment in Practice: The Newey-West Approach}

The alignment and the lag selection criteria depend on prior knowledge of spectral parameters. In practice, however, the parameters are not known. As in Newey and West (1994), one solution is to estimate the parameters using a truncated kernel estimator and a thumb rule for bandwidth selection. The parameter estimates are then plugged into Eqs. (8) and (9).

The truncated kernel cannot be used however in what follows, because it can generate negative spectral estimates. Consider therefore the following analogue Bartlett kernel estimators of (3), (6) and (7):

$$
\begin{array}{r}
\hat{s}_{x y}^{(1)}(m, a)=\frac{1}{2 \pi} \sum_{n=-m}^{m} \kappa(n, m)|n| \hat{\gamma}_{x y}(a+n), \quad \hat{s}_{x x}^{(1)}(m)=\frac{1}{2 \pi} \sum_{n=-m}^{m} \kappa(n, m)|n| \hat{\gamma}_{x x}(n), \\
\hat{s}_{y y}^{(1)}(m)=\frac{1}{2 \pi} \sum_{n=-m}^{m} \kappa(n, m)|n| \hat{\gamma}_{y y}(n), \quad \hat{s}_{x x}(m)=\frac{1}{2 \pi} \sum_{n=-m}^{m} \kappa(n, m) \hat{\gamma}_{x x}(n), \\
\hat{s}_{y y}(m)=\frac{1}{2 \pi} \sum_{n=-m}^{m} \kappa(n, m) \hat{\gamma}_{y y}(n), \text { with } m=\left\lceil\zeta(T / 100)^{1 / 5}\right\rceil,
\end{array}
$$

where, following Hirukawa (2010), and as shown in "Appendix 5", the thumb rule for the plug-in bandwidth $m$ has an optimal growth rate of $O\left(T^{1 / 5}\right)$, and where $\zeta$ is a positive constant. Monte Carlo experiments presented in previous articles, such as Newey and West (1994), typically suggest values of $\zeta$ ranging from 3 to 12.

The alignment parameter $a^{*}$ is estimated using an analogue representation of (9),

$$
\hat{a}=\underset{a_{\min } \leq a \leq a_{\max }}{\arg \min } \sum_{n=a_{\min }}^{a_{\max }}|n-a| \cdot\left|\hat{\gamma}_{x y}(n)\right|,
$$

and the following parameters are estimated conditionally on $m$ and $\hat{a}$ :

$$
\begin{gathered}
\hat{\Lambda}_{m, a}=\frac{\hat{\sigma}_{X Y}(m, \hat{a})}{\sqrt{\hat{\sigma}_{X X}(m) \hat{\sigma}_{Y Y}(m)},} \\
\hat{\sigma}_{X Y}(m, \hat{a})= \begin{cases}\sum_{t=m+\hat{a}}^{T} \frac{\left[\left(1-L^{m}\right) X_{t}-m \bar{\mu}_{x}\right]\left[\left(1-L^{m}\right) Y_{t-\hat{a}}-m \bar{\mu}_{y}\right]}{T-m-\hat{a}}, & \hat{a} \geq 0, \\
\sum_{t=m-\hat{a}}^{T} \frac{\left[\left(1-L^{m}\right) X_{t+\hat{a}}-m \bar{\mu}_{x}\right]\left[\left(1-L^{m}\right) Y_{t}-m \bar{\mu}_{y}\right]}{T-m+\hat{a}} & \hat{a}<0,\end{cases}
\end{gathered}
$$


and

$$
\hat{\psi}_{m, a}=\frac{\hat{s}_{x y}^{(1)}(m, \hat{a})}{\sqrt{\hat{s}_{x x}(m) \hat{s}_{y y}(m)}}-\frac{\hat{\Lambda}_{m, a}}{2}\left(\frac{\hat{s}_{x x}^{(1)}(m)}{\hat{s}_{x x}(m)}+\frac{\hat{s}_{y y}^{(1)}(m)}{\hat{s}_{y y}(m)}\right),
$$

which, when plugged into the analogue version of Eq. (8), lead to

$$
\hat{k}=\left\lceil 1.4422\left[\left(\frac{\hat{\psi}_{m, a}}{1-\hat{\Lambda}_{m, a}^{2}}\right)^{2} T\right]^{\frac{1}{3}}\right]
$$

and hence to

$$
\hat{\Lambda}_{k, a}=\frac{\hat{\sigma}_{X Y}(\hat{k}, \hat{a})}{\sqrt{\hat{\sigma}_{X X}(\hat{k}) \hat{\sigma}_{Y Y}(\hat{k})}} .
$$

\section{Lag Selection and Alignment in Practice: The Andrews-Monahan Approach}

Data can be prewhitened before the procedure of this article is employed, and the results can be recolored afterwards. The use of prewhitening may improve the estimator performance in some cases; see for example Andrews and Monahan (1992) and Newey and West (1994) for details and implementation.

Notice that prewhitening precludes the direct application of the correlation block estimator to the data, in which case the procedure may lose attractiveness to practitioners that feel more comfortable when periodicity information is provided by the optimal choice of the time interval $k^{*}$ based on data that has not been prewhitened.

Andrews (1991) shows how parametric estimates can be used to obtain the first-step spectral parameters, while Andrews and Monahan (1992) explain how the procedure can be improved by using prewhitening. Consider for example a generalization of Andrews and Monahan (1992) Monte Carlo procedure, where here a VAR $(p)$ is used instead of a VAR (1) for prewhitening, and a VAR (1) is used instead of an AR (1) for the first-step spectral parameter estimation. Prewhitening employs a VAR $(p)$ estimated using LS:

$$
\left[\begin{array}{l}
x_{t} \\
y_{t}
\end{array}\right]=\tilde{\boldsymbol{B}}_{0}+\sum_{n=1}^{p} \tilde{\boldsymbol{B}}_{n}\left[\begin{array}{c}
x_{t-n} \\
y_{t-n}
\end{array}\right]+\left[\begin{array}{c}
\tilde{x}_{t}^{w} \\
\tilde{y}_{t}^{w}
\end{array}\right]
$$

where $\tilde{x}_{t}^{w}$ and $\tilde{y}_{t}^{w}$ are the prewhitened series. A VAR (1),

$$
\left[\begin{array}{c}
\tilde{x}_{t}^{w} \\
\tilde{y}_{t}^{w}
\end{array}\right]=\tilde{\boldsymbol{C}}_{0}+\tilde{\boldsymbol{C}}_{1}\left[\begin{array}{c}
\tilde{x}_{t-1}^{w} \\
\tilde{y}_{t-1}^{w}
\end{array}\right]+\left[\begin{array}{c}
\tilde{\varepsilon}_{t} \\
\tilde{v}_{t}
\end{array}\right]
$$


with an estimated innovation covariance matrix $\tilde{\Sigma}$, can now be used to estimate the first-step parameters, leading to the following spectral matrix estimates:

$$
\tilde{\boldsymbol{S}}=\left[\begin{array}{cc}
\tilde{s}_{x x} & \tilde{s}_{x y} \\
\tilde{s}_{x y} & \tilde{s}_{y y}
\end{array}\right]=\frac{1}{2 \pi}\left(\boldsymbol{I}-\tilde{\boldsymbol{C}}_{1}\right)^{-1} \tilde{\Sigma}\left(\boldsymbol{I}-\tilde{\boldsymbol{C}}_{1}^{\prime}\right)^{-1}
$$

and smoothness matrix estimates:

$$
\tilde{\boldsymbol{S}}^{(1)}=\left[\begin{array}{cc}
\tilde{s}_{x x}^{(1)} & \tilde{s}_{x y}^{(1)} \\
\tilde{s}_{x y}^{(1)} & \tilde{s}_{y y}^{(1)}
\end{array}\right]=\frac{1}{2 \pi}\left(\tilde{\boldsymbol{H}}+\tilde{\boldsymbol{H}}^{\prime}\right), \quad \tilde{\boldsymbol{H}}=\left(\boldsymbol{I}-\tilde{\boldsymbol{C}}_{1}\right)^{-2} \tilde{\boldsymbol{C}}_{1} \sum_{n=0}^{\infty} \tilde{\boldsymbol{C}}_{1}^{n} \tilde{\Sigma}\left(\tilde{\boldsymbol{C}}_{1}^{\prime}\right)^{n},
$$

where, in practice, the summation is truncated when $n$ reaches a value that is deemed large enough. The optimal time interval based on the Andrews-Monahan approach is therefore given by

$$
\tilde{k}=\left\lceil 1.4422\left[\left(\frac{\tilde{\psi}}{1-\tilde{\lambda}^{2}}\right)^{2} T\right]^{\frac{1}{3}}\right]
$$

where

$$
\tilde{\lambda}=\frac{\tilde{s}_{x y}}{\sqrt{\tilde{s}_{x x} \tilde{s}_{y y}}} \text { and } \tilde{\psi}=\frac{\tilde{s}_{x y}^{(1)}}{\sqrt{\tilde{s}_{x x} \tilde{s}_{y y}}}-\frac{\tilde{\lambda}}{2}\left(\frac{\tilde{s}_{x x}^{(1)}}{\tilde{s}_{x x}}+\frac{\tilde{s}_{y y}^{(1)}}{\tilde{s}_{y y}}\right)
$$

The long-run covariance matrix estimate can now be recolored:

$$
\left[\begin{array}{c}
\tilde{\sigma}_{X X}(\tilde{k}) \\
\tilde{\sigma}_{X Y}(\tilde{k}) \\
\tilde{\sigma}_{X Y}(\tilde{k}) \\
\tilde{\sigma}_{Y Y}(\tilde{k})
\end{array}\right]=\tilde{\boldsymbol{Q}} \cdot\left[\begin{array}{c}
\tilde{\sigma}_{X^{w} X^{w}}(\tilde{k}) \\
\tilde{\sigma}_{X^{w} Y^{w}}(\tilde{k}) \\
\tilde{\sigma}^{w} Y^{w}(\tilde{k}) \\
\tilde{\sigma}_{Y^{w} Y^{w}}(\tilde{k})
\end{array}\right] \cdot \boldsymbol{Q}^{\tilde{\prime}}
$$

where

$$
\tilde{\sigma}_{X^{w} Y^{w}}(\tilde{k})=\sum_{t=k}^{T} \frac{\left(1-L^{\tilde{k}}\right) \tilde{X}_{t}^{w}\left(1-L^{\tilde{k}}\right) \tilde{Y}_{t}^{w}}{T-\tilde{k}}, \quad \tilde{\boldsymbol{Q}}=\left(\boldsymbol{I}-\sum_{n=1}^{p} \tilde{\boldsymbol{B}}_{n}\right)^{-1}
$$

and where $\tilde{X}_{t}^{w}$ and $\tilde{Y}_{t}^{w}$ are the integrated versions of $\tilde{x}_{t}^{w}$ and $\tilde{y}_{t}^{w}$, leading to:

$$
\tilde{\Lambda}_{k}=\frac{\tilde{\sigma}_{X Y}(\tilde{k})}{\sqrt{\tilde{\sigma}_{X X}(\tilde{k}) \tilde{\sigma}_{Y Y}(\tilde{k})}}
$$




\section{Monte Carlo Simulations}

"Appendix 6" shows the results of Monte Carlo simulations with 10,000 iterations each, based on parameters estimated from Dow Jones Industrial Average returns data. Table 1 presents combinations of long-run correlation values $\lambda$ equal to $0.0,0.4$ and 0.8 (column 2) and of sample sizes $T$ equal to 100,400 and 1600 (column 3).

\section{Experiment}

Series $x_{t}$ and $y_{t}$ are generated using $\operatorname{VMA}(5)$ and $\operatorname{GARCH}(1,1)$ processes with independent and identically $t$-distributed innovations $v_{t}$ and $\xi_{t}$ :

$$
\begin{aligned}
& {\left[\begin{array}{c}
x_{t} \\
y_{t}
\end{array}\right]=\left[\begin{array}{cr}
1 & \alpha\left(1-\theta \sum_{n=1}^{5} \frac{L^{n}}{5}\right) \\
\alpha\left(1-\theta \sum_{n=1}^{5} \frac{L^{n}}{5}\right) & L^{3}
\end{array}\right]\left[\begin{array}{l}
v_{t} \\
\phi_{t}
\end{array}\right],} \\
& v_{t}=\delta_{0}+\chi_{t} \sqrt{g_{t}}, \quad g_{t}=\beta_{0}+\beta_{1}\left(v_{t-1}-\delta_{0}\right)^{2}+\beta_{2} g_{t-1}, \quad \operatorname{var}\left(\chi_{t}\right)=1 \text {, } \\
& \phi_{t}=\delta_{0}+\xi_{t} \sqrt{h_{t}}, \quad h_{t}=\beta_{0}+\beta_{1}\left(\phi_{t-1}-\delta_{0}\right)^{2}+\beta_{2} h_{t-1}, \quad \operatorname{var}\left(\xi_{t}\right)=1 \text {, }
\end{aligned}
$$

where parameters $\delta_{0}, \beta_{0}, \beta_{1}$, and $\beta_{2}$ and the number of degrees of freedom (DOF) of the $t$-distributed innovations were estimated from Dow Jones returns data ranging from January 9, 1990, to August 1, 2001, corresponding to 3018 daily observations. The estimated parameters are:

$$
\begin{aligned}
& \delta_{0}=\underset{(0.000133)}{0.000648}, \quad \beta_{0}=\underset{(2.26 E-07)}{6.42 E-07}, \quad \beta_{1}=\underset{(0.007509)}{0.050154}, \\
& \beta_{2}=\underset{(0.008042)}{0.944037} \text {, and } \mathrm{DOF}=\underset{(0.564310)}{5.605809},
\end{aligned}
$$

where the values between parentheses represent standard errors.

The values of $\alpha$ and $\theta$ determine the value of $\lambda$ according to the equation

$$
\lambda=\frac{2 \alpha(1-\theta)}{1+\alpha^{2}(1-\theta)^{2}} .
$$

The experiment creates pairs of series that emulate the Dow Jones statistical process, allowing however for different levels of long-run correlation. A lag of three periods is applied to series $y_{t}$ to test the effectiveness of the alignment criterion. Parameter $\theta$ assumes values of $0.0,0.5$ and 0.8 , as shown in column 1 , and is used to evaluate the sensitivity of the estimation procedures to the presence of small moving average terms at longer lags. This type of process is commonly found in economic and financial data and tends to pose problems to some estimators, as discussed for example in Cochrane (1988), Schwert (1989) and Newey and West (1994). 


\section{Benchmarks}

Four common estimation procedures are taken as benchmarks that represent current and common practices and are presented in columns 4-9 of Table 1:

(a) aggregation of daily data over time intervals of 5 and 20 days, roughly representing correlation estimates based on weekly and monthly aggregates (columns 4 and 5, "5 Days" and "20 Days", respectively);

(b) VAR estimation with order selection based on the Akaike information criterion (AIC) and on the Schwarz Bayesian criterion (SBC), with long-run correlation estimates calculated using the spectral matrix procedure described on page 836 of Andrews (1991) (columns 6 and 7, "AIC" and "SBC", respectively);

(c) block estimator using the Schwert (1989) lag selection thumb rule $k_{T R}=$ $\left\lceil 4(T / 100)^{1 / 4}\right\rceil$ (column 8, " $k_{T R}$ "); and

(d) block estimator of the covariance matrix using the Newey-West automatic lag selection, with standard weight vector of ones and without prewhitening (column 9, “ $k_{N W}$ ").

These benchmarks are compared to the following new estimators proposed in this article:

(e) the block estimator with automatic lag selection criterion and without alignment $\left(a^{*}=0\right)$ based on the Newey-West approach, as given by Eq. (11) (columns 10, 11 and 12, " $k_{2}$ ", " $k_{4}$ " and " $k_{12}$ ", respectively);

(f) the block estimator with automatic lag selection and alignment criteria based on the Newey-West approach, as given by Eqs. (10) and (11) (columns 13, 14 and 15 , " $k_{2, a}$ ", " $k_{4, a}$ " and " $k_{12, a}$ ", respectively); and

(g) the block estimator with prewhitening and automatic lag selection criterion using the Andrews-Monahan approach, given by Eq. (12) (columns 16, 17 and 18, " $k_{A, 1}$ ", " $k_{A, A I C}$ " and " $k_{A, S B C}$ ", respectively).

The estimators based on the Newey-West approach use the following first-step thumb rule:

$$
m=\left\lceil\zeta(T / 100)^{1 / 5}\right\rceil
$$

with values of $\zeta$ equal to 2,4 and 12 . The prewhitening step of the estimators based on the Andrews-Monahan approach uses VAR orders equal to one or selected per the Akaike information criterion (AIC) and the Schwarz Bayesian criterion (SBC).

\section{Monte Carlo Results: Benchmarks}

Table 1 depicts the MSE values for each estimator and different combinations of $\theta, \lambda$ and $T$, where

$$
\operatorname{MSE}=(\overline{\hat{\Lambda}}-\lambda)^{2}+\hat{\sigma}_{\Lambda}^{2}, \quad \overline{\hat{\Lambda}}-\lambda=\frac{1}{10000} \sum_{n=1}^{10000} \hat{\Lambda}_{n}-\lambda \text { and } \quad \hat{\sigma}_{\Lambda}^{2}=\frac{1}{10000} \sum_{n=1}^{10000}\left(\hat{\Lambda}_{n}-\overline{\hat{\Lambda}}\right)^{2}
$$


The results in columns 4 and 5 in Table 1 ("5 Days" and "20 Days") indicate that the common practice of aggregating high-frequency data into longer time intervals may lead to poor MSE statistics. As expected, estimators based on aggregate weekly data (column 4) perform poorly for high values of $\lambda$. Estimators based on aggregate monthly data (column 5), on the other hand, perform poorly for small samples, due to the wasteful use of the available information. The simulations indicate that this practice should be avoided.

Column 6 ("AIC") shows that the parametric VAR estimator that uses the Akaike information criterion for order selection performs poorly for small sample sizes. Column 7 ("SBC"), in contrast, reveals that the VAR estimator that use the Schwartz Bayesian criterion for order selection tends to perform better than other benchmarks, but not as well as this article's proposed estimator. For example, for $\theta=0.8, \lambda=0.8$, and $T=400$, the VAR-SBC estimator has a MSE of 0.175, which is 3.6 times higher than the MSE of 0.048 of the proposed estimator in column 14, and 5.3 times higher than the MSE of 0.033 of the proposed estimator in column 15.

Column 8 (" $k_{T R}$ ") gives the performance of the block estimator using the Schwert (1989) lag selection rule. This thumb rule performs poorly for high values of $\lambda$, as expected. Column 9 (" $k_{N W}$ ") presents the results for the covariance matrix estimator using the Newey and West (1994) automatic lag selection criterion. As in the case of the Schwert lag selection rule, this estimator performs poorly for high values of $\lambda$. The Newey-West criterion should not be used therefore for long-run correlation estimation through the estimation of the covariance matrix, in agreement with the results of "Nonparametric Estimation of Long-Run Correlation".

Columns 10, 11 and 12 (" $k_{2}$ ", " $k_{4}$ " and " $k_{12}$ ") show the results for the proposed block estimator using the lag selection criterion based on the Newey-West approach and without alignment $\left(a^{*}=0\right)$, as shown in "Nonparametric Estimation of Long-Run Correlation". Notice the clear improvement over the two previous benchmark block estimators (columns 8 and 9, " $k_{T R}$ " and " $k_{N W}$ "), particularly when parameter $\zeta$ is equal to 12 . The VAR-SBC estimator (column 7) however outperforms the proposed estimator without alignment in most cases, even when $\zeta$ is equal to 12 .

The proposed block estimator with automatic lag selection and alignment criteria based on the Newey-West approach, as described in "Nonparametric Estimation of Long-Run Correlation", outperforms all other estimators, as shown in column 13, 14

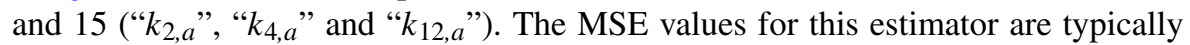
among the lowest for all combinations of parameters, and particularly when $\zeta$ is equal to 4 and 12. A choice of $\zeta$ equal to 2 or 4 tends to produce the best results when the values of $\theta$ are small, while a choice of $\zeta$ equal to 12 tends to be beneficial when the values of $\theta$ are large.

Finally, the results for the proposed block estimator based on the Andrews-Monahan approach described in "Nonparametric Estimation of Long-Run Correlation" are shown in columns 16,17 and 18 (" $k_{A, 1}$ ", " $k_{A, A I C}$ ", and " $k_{A, S B C}$ "). This estimator performs at par with the VAR-AIC and VAR-SBC, except for the VAR (1) case (" $k_{a, 1}$ "), which performs poorly. The latter is the result of omitted variable bias due to the misspecification of the model used in the prewhitening step. Notice also that this variant of the estimator performs well when $\lambda=0$ not for its own merits but simply because, in this case, there is no misspecification and the model is parsimonious. 
The Monte Carlo simulations reveal therefore that the joint use of the lag selection and alignment criteria based on the Newey-West approach is effective when processes contain small moving average terms at longer lags and possible time misalignments, without producing significant drawbacks. The proposed procedure leads to significant improvements over methods currently employed, suggesting that the estimator presented in this article should be considered a useful addition to the practitioner's toolbox.

\section{Monte Carlo Results: Spectral and Block Estimators}

As discussed in "Long-Run Correlation", a Monte Carlo experiment is also performed under the same Monte Carlo framework of the previous subsection to compare the finite sample performances of the Bartlett kernel spectral estimator of long-run correlation $\hat{\lambda}_{k}$ defined in (4) and of the block estimator of long-run correlation $\hat{\Lambda}_{k}$ defined in (5), both estimators using the same optimal lag and alignment procedures described in item (f) above.

Mean MSE values for the Bartlett kernel spectral estimator were found to be equal to 0.048 (" $k_{2, a}$ "), 0.035 (" $k_{4, a}$ "), and 0.033 (" $k_{12, a}$ "), compared to the to the mean MSE values for the block estimator of 0.049 (" $k_{2, a}$ "), 0.036 (" $k_{4, a}$ "), and 0.034 (" $k_{12, a}$ ") shown in column 13, 14 and 15 of Table 1. The Monte Carlo simulations confirm that both estimators have almost identical performances for all sample sizes and parameter choices. The Bartlett kernel spectral estimator produces slightly better MSE values for smaller sample sizes, while the block estimator produces slightly better MSE values for larger sample sizes.

As discussed in "Introduction", the interpretation of the block estimator is more intuitive and straightforward to practitioners who are not trained in spectral methods, therefore the preference in this article is given to this latter estimator.

\section{Conclusions}

Long-run correlation estimators have many applications in finance and economics, for example, in the study of stock returns and in the measurement of the relations and lags between monetary and real variables.

This paper used the approaches of Andrews and Monahan (1992) and Newey and West (1994) to develop automatic lag selection criteria for a nonparametric consistent long-run correlation estimator based on the block estimator ( $k$-lag difference correlation estimator). In addition, an alignment criterion that potentially enhances finite sample performance was presented.

A Monte Carlo experiment showed that the lag selection and the alignment criteria presented here are effective can be superior to commonly employed methods, such as aggregation over arbitrary time intervals, parametric VAR estimation, and Newey-West automatic lag selection of the covariance matrix.

The optimal yet unobtrusive long-run correlation estimator presented in this article intends to reduce the gap between econometric theory and practice by offering not only an asymptotically optimal alternative to current practices, but also a formal statistical framework for researchers dealing with time-series correlation studies. 
Acknowledgements Thanks to F. Araújo, S. Durlauf, J. Faria, B. Hansen, M. Hirukawa, M. Horvath, Y. Kitamura, B. Pötscher, B. Tabak, K. D. West, and anonymous referees for helpful comments and suggestions. This work was supported by the French National Research Agency Grant ANR-17-EURE-0020.

\section{Appendix 1}

The $k$-lag difference covariance estimator of $x_{t}$ and $y_{t}$ is:

$$
\hat{\sigma}_{X Y}(k)=\frac{1}{T-k} \sum_{t=k}^{T}\left[\left(1-L^{k}\right) X_{t}-k \bar{\mu}_{x}\right]\left[\left(1-L^{k}\right) Y_{t}-k \bar{\mu}_{y}\right]
$$

where $\left(1-L^{k}\right) X_{t}=X_{t}-X_{t-k}$ and $\left(1-L^{k}\right) Y_{t}=Y_{t}-Y_{t-k}$, such that

$$
\hat{\sigma}_{X Y}(k)=\frac{1}{T-k} \sum_{t=k}^{T}\left[\left(\sum_{i=1}^{k} x_{t-k+i}^{\prime}\right)\left(\sum_{j=1}^{k} y_{t-k+j}^{\prime}\right)\right],
$$

where $x_{t}^{\prime}=x_{t}-\bar{\mu}_{x}$ and $y_{t}^{\prime}=y_{t}-\bar{\mu}_{y}$, or

$$
\begin{aligned}
\hat{\sigma}_{X Y}(k)= & \frac{1}{T-k} \sum_{t=k}^{T}\left[\sum_{l=1}^{k-1}\left(\sum_{i=1}^{l} x_{t-k+i}^{\prime} y_{t-l+i}^{\prime}+\sum_{j=1}^{l} x_{t-l+j}^{\prime} y_{t-k+j}^{\prime}\right)+\sum_{m=1}^{k} x_{t-k+m}^{\prime} y_{t-k+m}^{\prime}\right] \\
= & \frac{1}{T-k}\left(\sum_{t=k}^{T} x_{t-k+1}^{\prime} y_{t}^{\prime}+2 \sum_{t=k-1}^{T} x_{t-k+2}^{\prime} y_{t}^{\prime}+\cdots+(k-1) \sum_{t=2}^{T} x_{t-1}^{\prime} y_{t}^{\prime}+k \sum_{t=1}^{T} x_{t}^{\prime} y_{t}^{\prime}\right. \\
& \left.+(k-1) \sum_{t=2}^{T} x_{t}^{\prime} y_{t-1}^{\prime}+\cdots+2 \sum_{t=k-1}^{T} x_{t}^{\prime} y_{t-k+2}^{\prime}+\sum_{t=k}^{T} x_{t}^{\prime} y_{t-k+1}^{\prime}\right)+R(T, k),
\end{aligned}
$$

where

$$
\begin{aligned}
R(T, k)= & -\frac{1}{T-k}\left[\sum _ { i = 2 } ^ { k - 1 } \sum _ { j = 1 } ^ { i - 1 } ( i - j ) \left(x_{j}^{\prime} y_{k-i+j}^{\prime}+x_{T-k+i-j+1}^{\prime} y_{T-j+1}^{\prime}\right.\right. \\
& \left.\left.+x_{k-i+j}^{\prime} y_{j}^{\prime}+x_{T-j+1}^{\prime} y_{T-k+i-j+1}^{\prime}\right)+\sum_{j=1}^{k-1}(k-j)\left(x_{j}^{\prime} y_{j}^{\prime}+x_{T-j+1}^{\prime} y_{T-j+1}^{\prime}\right)\right],
\end{aligned}
$$

or

$$
\frac{\hat{\sigma}_{X Y}(k)}{k}=\frac{T}{T-k} 2 \pi \hat{s}_{x y}(k)+\frac{R(T, k)}{k},
$$

where $\hat{s}_{x y}(k)$ is the Bartlett kernel estimate of the cross-spectrum at frequency zero with bandwidth $b=k$, and where the Bartlett kernel is defined as: 


$$
\kappa_{B}(n, b)= \begin{cases}1-|n| / b, & |n|<b, \\ 0, & |n| \geq b .\end{cases}
$$

Given the assumption of summable autocovariances and covariances, it follows that $R(T, k) / k=O_{p}(k / T)$, therefore, the block covariance estimator is asymptotically equivalent to $2 \pi$ times the Bartlett kernel estimator of the cross-spectrum at frequency zero with $b=k$ :

$$
\frac{\hat{\sigma}_{X Y}(k)}{k}=\frac{T}{T-k} 2 \pi \hat{s}_{x y}(k)+O_{p}\left(\frac{k}{T}\right) .
$$

\section{Appendix 2}

Hannan (1970, pg. 280), Priestley (1981, pg. 699) and Brockwell and Davis (1991, pg. 446) show that, under the consistency assumptions mentioned in "Nonparametric Estimation of Long-Run Correlation", in particular,

$$
\lim _{T \rightarrow \infty} k(T)=\infty \text { and } \lim _{T \rightarrow \infty} k(T) / T=0,
$$

the asymptotic covariance (Acov) between two spectra or cross-spectra Bartlett kernel estimators at frequency zero is

$$
\operatorname{Acov}\left(\hat{s}_{a b}(k), \hat{s}_{c d}(k)\right)=\frac{2}{3} \frac{k}{T}\left(s_{a c} s_{b d}+s_{a d} s_{b c}\right) .
$$

The long-run correlation and its estimator are defined as

$$
\begin{gathered}
\lambda=\frac{s_{x y}}{\sqrt{s_{x x} s_{y y}}}, \\
\hat{\lambda}_{k}=\frac{\hat{s}_{x y}(k)}{\sqrt{\hat{s}_{x x}(k) \hat{s}_{y y}(k)}} .
\end{gathered}
$$

Per (13), the components of (15) have an asymptotic covariance matrix given by

$$
\operatorname{Acov}(\hat{\boldsymbol{s}}(k))=\operatorname{cov}\left[\begin{array}{c}
\hat{s}_{x x}(k) \\
\hat{s}_{y y}(k) \\
\hat{s}_{x y}(k)
\end{array}\right]=\frac{2}{3} \frac{k}{T}\left[\begin{array}{ccc}
2 s_{x x}^{2} & 2 s_{x y}^{2} & 2 s_{x x} s_{x y} \\
2 s_{x y}^{2} & 2 s_{y y}^{2} & 2 s_{y y} s_{x y} \\
2 s_{x x} s_{x y} & 2 s_{y y} s_{x y} & s_{x x} s_{y y}+s_{x y}^{2}
\end{array}\right] .
$$

As in Hannan (1970, pg. 287), a Taylor expansion of (14) around $E\left[\hat{s}_{x x}(k)\right], E$ $\left[\hat{s}_{y y}(k)\right]$, and $E\left[\hat{s}_{x y}(k)\right]$ leads to

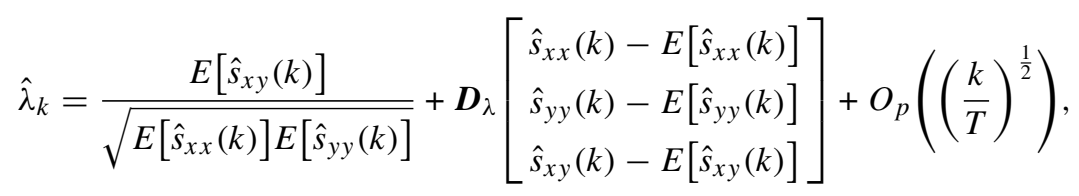


where

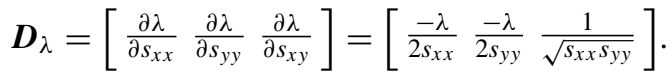

From (16), and since

$$
E\left[\hat{\lambda}_{k}\right]-E\left[\hat{s}_{x y}(k)\right] / \sqrt{E\left[\hat{s}_{x x}(k)\right] E\left[\hat{s}_{y y}(k)\right]}=O(k / T),
$$

one can conclude that the asymptotic variance of the long-run correlation estimator is given by

$$
\operatorname{Avar}\left(\hat{\lambda}_{k}\right)=\boldsymbol{D}_{\lambda} \cdot \operatorname{Acov}(\hat{\boldsymbol{s}}(k)) \cdot \boldsymbol{D}_{\lambda}^{\prime},
$$

such that

$$
\operatorname{Avar}\left(\hat{\lambda}_{k}\right)=\frac{2}{3} \frac{k}{T}\left(1-\lambda^{2}\right)^{2}
$$

\section{Appendix 3}

The long-run correlation asymptotic bias (Abias) is given by

$$
\operatorname{Abias}\left(\hat{\lambda}_{k}\right)=E\left[\hat{\lambda}_{k}-\lambda\right]=E\left[\frac{\hat{s}_{x y}(k)}{\sqrt{\hat{s}_{x x}(k) \hat{s}_{y y}(k)}}-\frac{s_{x y}}{\sqrt{s_{x x} s_{y y}}}\right] \text {. }
$$

Following Hannan (1970, pg. 283), and under the same consistency assumptions of "Appendix 2", the asymptotic bias vector is:

$$
\operatorname{Abias}(\hat{\boldsymbol{s}})=\operatorname{bias}\left[\hat{s}_{x x}(k) \hat{s}_{y y}(k) \hat{s}_{x y}(k)\right]^{\prime}=\frac{-1}{k}\left[s_{x x}^{(1)} s_{y y}^{(1)} s_{x y}^{(1)}\right]^{\prime},
$$

where

$$
s_{x x}^{(1)}=\frac{1}{2 \pi} \sum_{n=-\infty}^{\infty}|n| \gamma_{x x}(n), \quad s_{y y}^{(1)}=\frac{1}{2 \pi} \sum_{n=-\infty}^{\infty}|n| \gamma_{y y}(n) \text {, and } s_{x y}^{(1)}=\frac{1}{2 \pi} \sum_{n=-\infty}^{\infty}|n| \gamma_{x y}(n) \text {. }
$$

A Taylor expansion of (14) around $s_{x x}, s_{y y}$, and $s_{x y}$, with $T \rightarrow \infty$, leads to

$$
E\left[\hat{\lambda}_{k}-\lambda\right]=\boldsymbol{D}_{\lambda}\left[\begin{array}{c}
E\left[\hat{s}_{x x}(k)-s_{x x}\right] \\
E\left[\hat{s}_{y y}(k)-s_{y y}\right] \\
E\left[\hat{s}_{x y}(k)-s_{x y}\right]
\end{array}\right]+O\left(\frac{1}{k^{2}}\right)
$$

Therefore, the asymptotic bias of the long-run correlation estimator is

$$
\operatorname{Abias}\left(\hat{\lambda}_{k}\right)=\boldsymbol{D}_{\lambda} \cdot \operatorname{Abias}[\hat{\boldsymbol{s}}(k)],
$$


where vector $\mathbf{D}_{\lambda}$ is defined as in "Appendix 2", implying that

$$
\operatorname{Abias}\left(\hat{\lambda}_{k}\right)=-\frac{\psi}{k}, \quad \psi=\frac{s_{x y}^{(1)}}{\sqrt{s_{x x} s_{y y}}}-\frac{\lambda}{2}\left(\frac{s_{x x}^{(1)}}{s_{x x}}+\frac{s_{y y}^{(1)}}{s_{y y}}\right) .
$$

\section{Appendix 4}

Problem: to find the optimal lag selection $k^{*}$ that minimizes the asymptotic mean square error (AMSE) of the long-run correlation estimator,

$$
\operatorname{AMSE}\left(\hat{\lambda}_{k}\right)=\left[\operatorname{Abias}\left(\hat{\lambda}_{k}\right)\right]^{2}+\operatorname{Avar}\left(\hat{\lambda}_{k}\right)
$$

From (17)-(19), and under the same consistency assumptions of “Appendix 2", it is straightforward to see that the AMSE is given by

$$
\operatorname{AMSE}\left(\hat{\lambda}_{k}\right)=\frac{1}{k^{2}} \psi^{2}+\frac{k}{T} \frac{2}{3}\left(1-\lambda^{2}\right)^{2}
$$

and consequently the optimal $k$ that minimizes the AMSE is

$$
k^{*}=\left\lceil 1.4422\left[\left(\frac{\psi}{1-\lambda^{2}}\right)^{2} T\right]^{\frac{1}{3}}\right\rceil
$$

where $\lceil\cdot\rceil$ represents the integer ceiling function.

\section{Appendix 5}

The problem is to find $m$ that minimizes:

$$
\operatorname{AMSE}\left(\hat{\eta}_{m}\right)=\left[\operatorname{Abias}\left(\hat{\eta}_{m}\right)\right]^{2}+\operatorname{Avar}\left(\hat{\eta}_{m}\right)
$$

where

$$
\hat{\eta}_{m}=\frac{\hat{\psi}_{m}}{1-\hat{\lambda}_{m}^{2}}, \text { and } \eta=\frac{\psi}{1-\lambda^{2}}
$$

Following Hirukawa (2010) and Priestley (1981, pg. 325), the asymptotic covariance matrix of the spectral components of $\hat{\eta}_{m}$ is given by 


$$
\operatorname{Acov}(\hat{\boldsymbol{s}}(m))=\frac{4 m^{3}}{3 T}\left[\begin{array}{llllll}
\frac{s_{x x}^{2}}{m^{2}} & \frac{s_{x y}^{2}}{m^{2}} & \frac{s_{x x} s_{x y}}{m^{2}} & \frac{s_{x x}^{2}}{4 m} & \frac{s_{x y}^{2}}{4 m} & \frac{s_{x x} s_{x y}}{4 m} \\
\frac{s_{x y}^{2}}{m^{2}} & \frac{s_{y y}^{2}}{m^{2}} & \frac{s_{y y} s_{x y}}{m^{2}} & \frac{s_{x y}^{2}}{4 m} & \frac{s_{y y}^{2}}{4 m} & \frac{s_{y y} s_{x y}}{4 m} \\
\frac{s_{x x} s_{x y}}{m^{2}} & \frac{s_{y y} s_{x y}}{m^{2}} & \frac{s_{x x} s_{y y}+s_{x y}^{2}}{2 m^{2}} & \frac{s_{x x} s_{x y}}{4 m} & \frac{s_{y y} s_{x y}}{4 m} & \frac{s_{x x} s_{y y}+s_{x y}^{2}}{8 m} \\
\frac{s_{x x}^{2}}{4 m} & \frac{s_{x y}^{2}}{4 m} & \frac{s_{x x} s_{x y}}{4 m} & \frac{s_{x x}^{2}}{10} & \frac{s_{x y}^{2}}{10} & \frac{s_{x x} s_{x y}}{10} \\
\frac{s_{x y}^{2}}{4 m} & \frac{s_{y y}^{2}}{4 m} & \frac{s_{y y} s_{x y}}{4 m} & \frac{s_{x y}^{2}}{10} & \frac{s_{y y}^{2}}{10} & \frac{s_{y y} s_{x y}}{10} \\
\frac{s_{x x} s_{x y}}{4 m} & \frac{s_{y y} s_{x y}}{4 m} & \frac{s_{x x} s_{y y}+s_{x y}^{2}}{8 m} & \frac{s_{x x} s_{x y}}{10} & \frac{s_{y y} s_{x y}}{10} & \frac{s_{x x} s_{y y}+s_{x y}^{2}}{20}
\end{array}\right],
$$

where

$$
\hat{\boldsymbol{s}}(m)=\left[\hat{s}_{x x}(m) \hat{s}_{y y}(m) \hat{s}_{x y}(m) \hat{s}_{x x}^{(1)}(m) \hat{s}_{y y}^{(1)}(m) \hat{s}_{x y}^{(1)}(m)\right]^{\prime},
$$

and the asymptotic bias vector by

$$
\operatorname{Abias}(\hat{\boldsymbol{s}}(m))=\frac{-1}{m}\left[\begin{array}{llllll}
s_{x x}^{(1)} & s_{y y}^{(1)} & s_{x y}^{(1)} & s_{x x}^{(1)} & s_{y y}^{(1)^{2}} & s_{x y}^{(1)^{2}}
\end{array}\right]^{\prime} .
$$

As in "Appendix 2", a Taylor expansion of (22) around $E\left[\hat{s}_{x x}(m)\right], E\left[\hat{s}_{y y}(m)\right], E$ $\left[\hat{s}_{x y}(m)\right], E\left[\hat{s}_{x x}^{(1)}(m)\right], E\left[\hat{s}_{y y}^{(1)}(m)\right]$, and $E\left[\hat{s}_{x y}^{(1)}(m)\right]$ leads to

$$
\hat{\eta}_{m}=\hat{\eta}_{m}^{E}+\boldsymbol{D}_{\eta}\left[\begin{array}{c}
\hat{s}_{x x}(m)-E\left[\hat{s}_{x x}(m)\right] \\
\hat{s}_{y y}(m)-E\left[\hat{s}_{y y}(m)\right] \\
\hat{s}_{x y}(m)-E\left[\hat{s}_{x y}(m)\right] \\
\hat{s}_{x x}^{(1)}(m)-E\left[\hat{s}_{x x}^{(1)}(m)\right] \\
\hat{s}_{y y}^{(1)}(m)-E\left[\hat{s}_{y y}^{(1)}(m)\right] \\
\hat{s}_{x y}^{(1)}(m)-E\left[\hat{s}_{x y}^{(1)}(m)\right]
\end{array}\right]+O_{p}\left(\left(\frac{m^{3}}{T}\right)^{\frac{1}{2}}\right),
$$

where

$$
\begin{gathered}
\boldsymbol{D}_{\eta}=\left[\frac{\partial \eta}{\partial s_{x x}} \frac{\partial \eta}{\partial s_{y y}} \frac{\partial \eta}{\partial s_{x y}} \frac{\partial \eta}{\partial s_{x x}^{(1)}} \frac{\partial \eta}{\partial s_{x y}^{(1)}} \frac{\partial \eta}{\partial s_{x y}^{(1)}}\right] \\
\hat{\eta}_{m}^{E}=\frac{1}{1-\hat{\lambda}_{m}^{E}}\left[\frac{E\left[s_{x y}^{(1)}(m)\right]}{\sqrt{E\left[s_{x x}(m)\right] E\left[s_{y y}(m)\right]}}-\frac{\hat{\lambda}_{m}^{E}}{2}\left(\frac{E\left[s_{x x}^{(1)}(m)\right]}{E\left[s_{x x}(m)\right]}+\frac{E\left[s_{y y}^{(1)}(m)\right]}{E\left[s_{y y}(m)\right]}\right)\right],
\end{gathered}
$$

and

$$
\hat{\lambda}_{m}^{E}=\frac{E\left[\hat{s}_{x y}(m)\right]}{\sqrt{E\left[\hat{s}_{x x}(m)\right] E\left[\hat{s}_{y y}(m)\right]}} .
$$

From (23), and since 


$$
E\left[\hat{\eta}_{m}\right]-\hat{\eta}_{m}^{E}=O\left(m^{3} / T\right)
$$

one can conclude that the asymptotic variance of $\hat{\eta}_{m}$ is given by

$$
\operatorname{Avar}\left(\hat{\eta}_{m}\right)=\boldsymbol{D}_{\eta} \cdot \operatorname{Acov}(\hat{\boldsymbol{s}}(m)) \cdot \boldsymbol{D}_{\eta}^{\prime},
$$

and in this case, as $m \rightarrow \infty$ and $m / T \rightarrow 0$, the problem of calculating the asymptotic variance of $\hat{\eta}_{m}$ reduces to

$$
\operatorname{Avar}\left(\hat{\eta}_{m}\right)=\frac{4}{30} \frac{m^{3}}{T} \cdot \boldsymbol{d}_{\eta} \cdot\left[\begin{array}{ccc}
s_{x x}^{2} & s_{x y}^{2} & s_{x x} s_{x y} \\
s_{x y}^{2} & s_{y y}^{2} & s_{y y} s_{x y} \\
s_{x x} s_{x y} & s_{y y} s_{x y} & \left(s_{x x} s_{y y}+s_{x y}^{2}\right) / 2
\end{array}\right] \cdot \boldsymbol{d}_{\eta}^{\prime},
$$

where

$$
\boldsymbol{d}_{\eta}=\left[\frac{\partial \eta}{\partial s_{x x}^{(1)}} \frac{\partial \eta}{\partial s_{x y}^{(1)}} \frac{\partial \eta}{\partial s_{x y}^{(1)}}\right]=\frac{1}{1-\lambda^{2}}\left[\frac{\lambda}{2 s_{x x}} \frac{\lambda}{2 s_{y y}} \frac{1}{\sqrt{s_{x x} s_{y y}}}\right]
$$

such that

$$
\operatorname{Avar}\left(\hat{\eta}_{m}\right)=\frac{1}{15} \frac{m^{3}}{T}
$$

Now, for the asymptotic bias, a Taylor expansion of (22) around $s_{x x}, s_{y y}, s_{x y}, s_{x x}^{(1)}$, $s_{y y}^{(1)}$, and $s_{x y}^{(1)}$ leads to

$$
E\left[\hat{\eta}_{m}-\eta\right]=\boldsymbol{D}_{\eta}\left[\begin{array}{c}
E\left[\hat{s}_{x x}(m)-s_{x x}\right] \\
E\left[\hat{s}_{y y}(m)-s_{y y}\right] \\
E\left[\hat{s}_{x y}(m)-s_{x y}\right] \\
E\left[\hat{s}_{x x}^{(1)}(m)-s_{x x}^{(1)}\right] \\
E\left[\hat{s}_{y y}^{(1)}(m)-s_{y y}^{(1)}\right] \\
E\left[\hat{s}_{x y}^{(1)}(m)-s_{x y}^{(1)}\right]
\end{array}\right]+O\left(\frac{1}{m^{2}}\right),
$$

and therefore the asymptotic bias of $\hat{\eta}_{m}$ is

$$
\operatorname{Abias}\left(\hat{\eta}_{m}\right)=\boldsymbol{D}_{\eta} \cdot \operatorname{Abias}(\hat{\boldsymbol{s}}(m)),
$$

and the equation for the asymptotic bias can be summarized as:

$$
\operatorname{Abias}\left(\hat{\eta}_{m}\right)=\rho / m,
$$

where $\rho$ represents a trivial yet lengthy combination of parameters. 
Given equations (24) and (25), the solution to problem (21) is

$$
m=\left\lceil 10 \rho^{2} T\right\rceil^{1 / 5} .
$$

\section{Appendix 6}

See Table 1. 


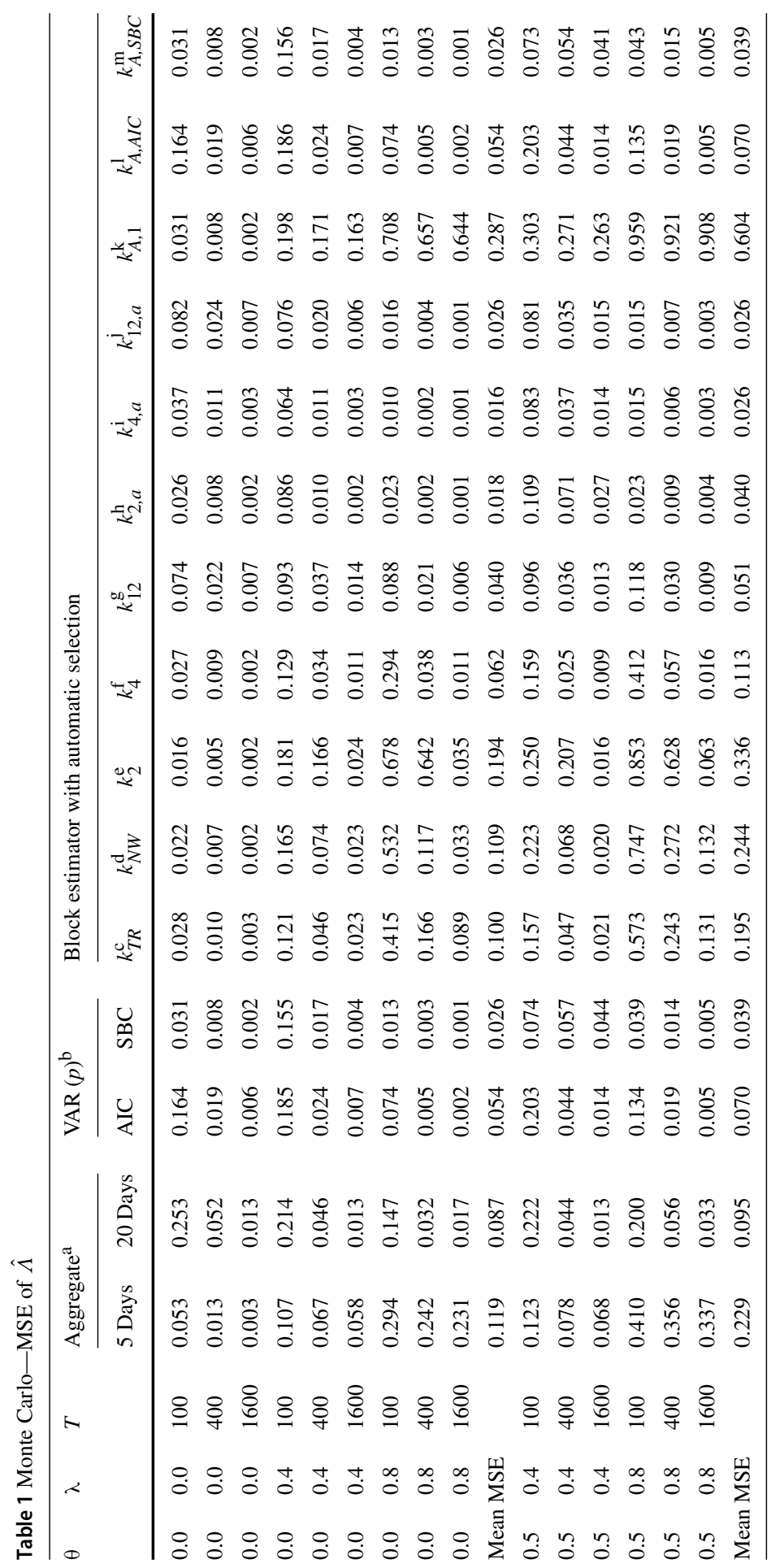




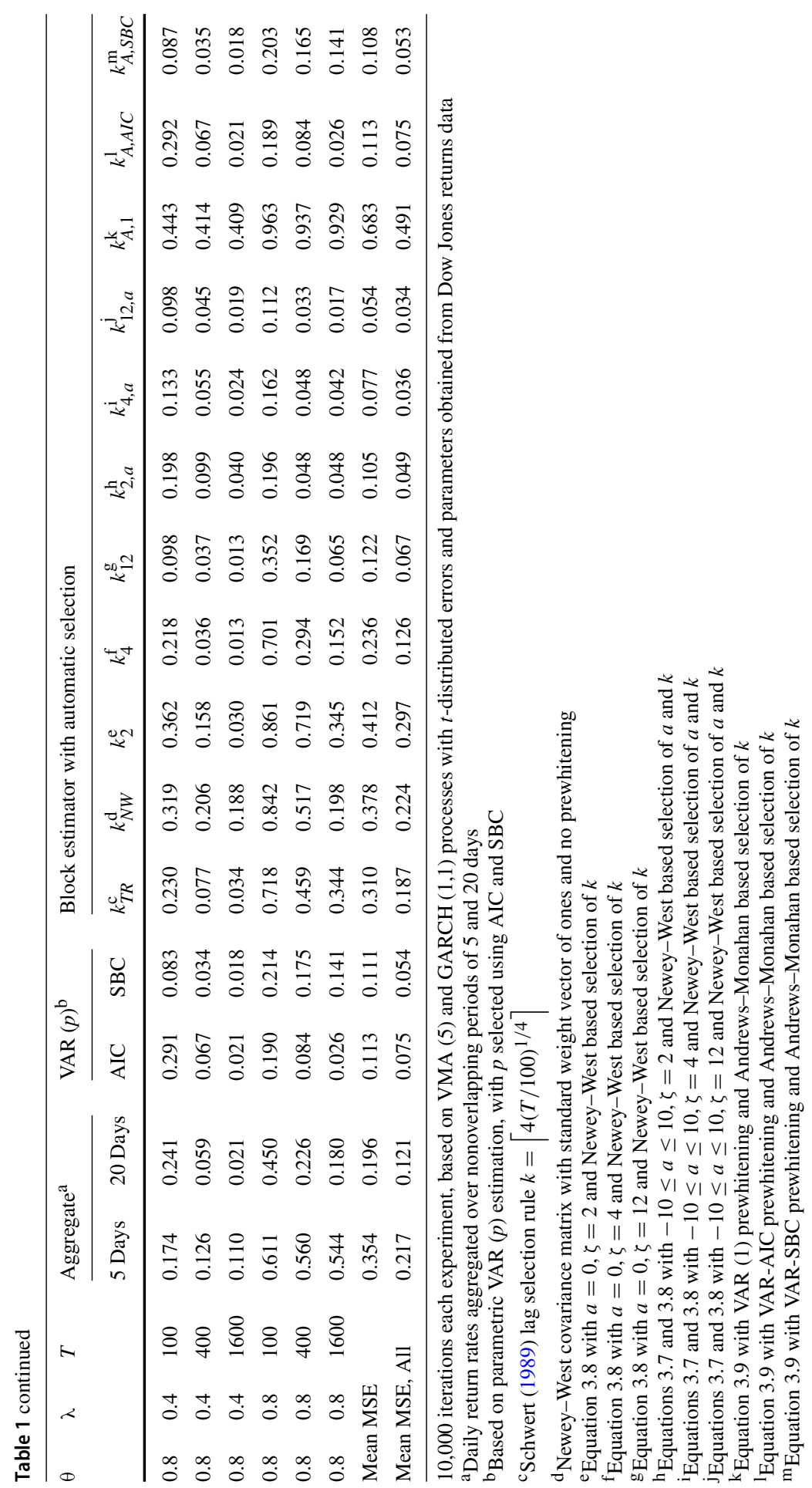




\section{References}

Ammer, J., and J. Mei. 1996. Measuring International Economic Linkages with International Stock Market Data. The Journal of Finance 51 (5): 1743-1763.

Anderson, T.W. 1971. The Statistical Analysis of Time Series. New York: Wiley.

Andrews, D.W.K. 1991. Heteroskedasticity and Autocorrelation Consistent Covariance Matrix Estimation. Econometrica 59 (3): 817-858.

Andrews, D.W.K., and J.C. Monahan. 1992. An Improved Heteroskedasticity and Autocorrelation Consistent Covariance Matrix Estimator. Econometrica 60 (4): 953-966.

Bartlett, M.S. 1950. Periodogram Analysis and Continuous Spectra. Biometrika 37 (1/2): 1-16.

Brockwell, P.J., and R.A. Davis. 1991. Time Series: Theory and Methods. New York: Springer.

Campbell, J.Y., and J. Ammer. 1993. What Moves the Stock and Bond Markets? A Variance Decomposition for Long-Term Asset Returns. The Journal of Finance 48 (1): 3-37.

Cochrane, J.H. 1988. How Big is the Random Walk in GNP? Journal of Political Economy 96: 893-920.

Cochrane, J.H., and A.M. Sbordone. 1988. Multivariate Estimates of the Permanent Components of GNP and Stock Prices. Journal of Economic Dynamics and Control 12: 255-296.

Diks, C., and V. Panchenko. 2006. A New Statistic and Practical Guidelines for Nonparametric Granger Causality Testing. Journal of Economic Dynamics \& Control 30: 1647-1669.

Engle, R.F., and C.W.J. Granger. 1987. Co-Integration and Error Correction: Representation, Estimation, and Testing. Econometrica 55: 251-276.

Fisher, M.E., and J.J. Seater. 1993. Long-Run Neutrality and Superneutrality in an ARIMA Framework. American Economic Review 83: 402-415.

Forbes, K., and R. Rigobon. 2002. No Contagion, Only Interdependence: Measuring Stock Market Comovements. The Journal of Finance 57 (5): 2223-2261.

Fuller, W.A. 1976. Introduction to Statistical Time Series. New York: Wiley.

Granger, C.W.J. 1969. Investigating Casual Relations by Econometric Models and Cross-Spectral Methods. Econometrica 37 (3): 424-438.

Granger, C.W.J., and R. Engle. 1983. Applications of Spectral Analysis in Econometrics. In Handbook of Statistics, vol. 3, ed. D.R. Brillinger and P.R. Krishnaiah. Amsterdam: Elsevier.

Granger, C.W.J., and J.-L. Lin. 1995. Causality in the Long Run. Econometric Theory 11: 530-536.

Granger, C.W.J., and H.J.B. Rees. 1968. Spectral Analysis of the Term Structure of the Interest Rates. Review of Economic Studies 35: 67-76.

Granger, C.W.J., and M.W. Watson. 1984. Time Series and Spectral Methods in Econometrics. In Handbook of Econometrics, vol. II, ed. Z. Griliches and M.D. Intriligator. New York: North-Holland.

Granger, C.W.J., and A.A. Weiss. 1983. Time Series Analysis of Error-Correction Models. Studies in Econometrics, Time Series, and Multivariate Statistics. New York: Academic Press.

Hannan, E.J. 1970. Multiple Time Series. New York: Wiley.

Hirukawa, M. 2010. A Two-Stage Plug-In Bandwidth Selection and Its Implementation for Covariance Estimation. Econometric Theory 26 (3): 710-743.

King, R.G., and M.W. Watson. 1994. The Post-War US Phillips Curve: A Revisionist Econometric History. In Carnegie-Rochester Conference Series on Public Policy, vol. 41, ed. A.H. Meltzer and C.I. Plosser. Amsterdam: North-Holland.

Koopmans, L.H. 1974. The Spectral Analysis of Time Series. San Diego: Academic Press.

McCallum, B.T. 1984. On Low-Frequency Estimates of Long-Run Relationships in Macroeconomics. Journal of Monetary Economics 14: 3-14.

McCallum, B.T. 1993. Unit Roots in Macroeconomic Time Series: Some Critical Issues. Federal Reserve Bank of Richmond Economic Quarterly 79: 13-43.

Nachane, D.M., R.M. Nadkarni, and A.V. Karnik. 1988. Co-integration and Causality Testing of the Energy_GDP Relationship: A Cross-Country Study. Applied Economics 20: 1511-1531.

Newey, W.K., and K.D. West. 1987. A Simple, Positive Semi-Definite, Heteroskedasticity and Autocorrelation Consistent Covariance Matrix. Econometrica 55: 703-708.

Newey, W.K., and K.D. West. 1994. Automatic Lag Selection in Covariance Matrix Estimation. Review of Economic Studies 61: 631-653.

Priestley, M.B. 1981. Spectral Analysis and Time Series. London: Academic Press.

Schwert, G.W. 1989. Tests for Unit Roots: A Monte Carlo Investigation. Journal of Business and Economic Statistics 7 (2): 147-159. 